\title{
New Organic Activators for the Enantioselective Reduction of Aromatic Imines with Trichlorosilane
}

\author{
Osamu Onomura, a Yoshimi Kouchi, a Fumiaki Iwasaki,b \\ and Yoshihiro Matsumura ${ }^{a}{ }^{*}$
}

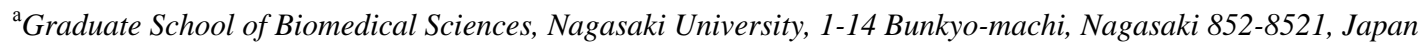 \\ ${ }^{\mathrm{b}}$ Tsukuba Research Laboratory, Tokuyama Corporation, 40 Wadai, Tsukuba 300-4247, Japan.
}

\begin{abstract}
N-Picolinoyl-(2S)-(diphenylhydroxymethyl)pyrrolidine was found to work as an organic activator in the reduction of aromatic imines to the corresponding amines by $\mathrm{Cl}_{3} \mathrm{SiH}$. The highest selectivity was $80 \%$ ee. This is the first data showing that $N$-formyl group is not always essential as $\mathrm{N}$-protecting group of pyrrolidine derivatives for the reduction of imines by $\mathrm{Cl}_{3} \mathrm{SiH}$.
\end{abstract}

Enantioselective reduction of ketones ${ }^{1}$ and imines $^{2}$ has been one of recent topics in asymmetric synthesis. ${ }^{3}$ A variety of reducing reagents have been used in the reductions but it is still worthwhile to exploit new methods which can be carried out using inexpensive reducing reagents under mild conditions. One of such reagents may be trichlorosilane $\left(\mathrm{Cl}_{3} \mathrm{SiH}\right)$, a liquid material easily available from silicon industry, ${ }^{4}$ though some activator is necessary for $\mathrm{Cl}_{3} \mathrm{SiH}$ to efficiently reduce ketones and imines. ${ }^{5} \mathrm{We}$ already reported chiral $\mathrm{N}$-formylpyrrolidine derivatives $\mathbf{1}$ as organic activators in the enantioselective reduction of ketones ${ }^{6}$ and imines ${ }^{7}$ with $\mathrm{Cl}_{3} \mathrm{SiH}$. The reduction proceeds smoothly at room temperature with good yields and enantioselectivity of up to $43 \%$ ee for the reduction of ketones and $66 \%$ ee for the reduction of imines. Recently, a new activator 2 for $\mathrm{Cl}_{3} \mathrm{SiH}$ in reducing imines with high enantioselectivity (up to $92 \%$ ee) was reported. ${ }^{8}$ The noticeable point in those reductions was that the presence of $N$-formyl substituent was essential for those reductions. In our continuing effort to exploit new chiral organic compounds in place of $\mathbf{1}$ to activate $\mathrm{Cl}_{3} \mathrm{SiH}^{9}$, we found $\mathrm{N}$-picolinoylpyrrolidine derivatives 3a-f to also work as organic activators in the reduction of aromatic imine 4 to amine 5 (Eq 1). This is the first data showing that $\mathrm{N}$-formyl group is not always essential in the structure of organic activators for $\mathrm{Cl}_{3} \mathrm{SiH}^{10,11}$

Key words: Organocatalysis; Asymmetric reduction; Imines; Optically active amines

* Corresponding author, Tel +81-95-819-2429, Fax +81-95-819-2476, E-mail matumura@net.nagasaki-u.ac.jp (Y. Matsumura). 
<smiles>[R]C1CCCN1C=O</smiles>

1a-e
a; $\mathrm{R}=\mathrm{H}$
b; $\mathrm{R}=\mathrm{CO}_{2} \mathrm{Me}$
c; $\mathrm{R}=\mathrm{CONHPh}$<smiles>[R]C1CCCN1C(=O)c1ccccn1</smiles>

3a-f

d; $\mathrm{R}=$ CONHNaph-1

e; $\mathrm{R}=\mathrm{CPh}_{2} \mathrm{OH}$

f; $\mathrm{R}=\mathrm{CHPh}_{2}$<smiles>CC(C)C(C(=O)Nc1ccccc1)N(C)C=O</smiles>

2

Figure 2. Chiral organic activator for reduction of imines by $\mathrm{Cl}_{3} \mathrm{SiH}$

Figure 1. Chiral organic activators for $\mathrm{Cl}_{3} \mathrm{SiH}$

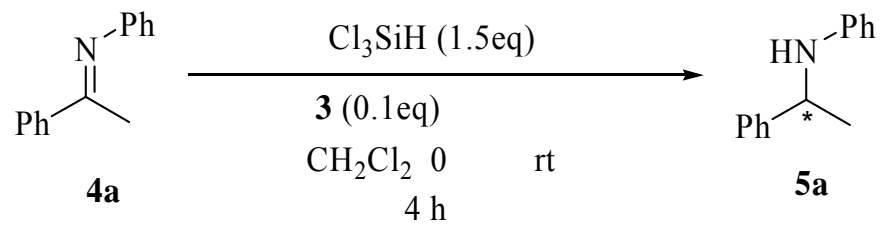

A typical reaction was carried out as follows. $\mathrm{Cl}_{3} \mathrm{SiH}(0.45 \mathrm{mmol})$ was added into a solution of $\mathbf{4 a}(0.3 \mathrm{mmol})$ and $\mathbf{3 a}(0.03 \mathrm{mmol})$ in $\mathrm{CH}_{2} \mathrm{Cl}_{2}(1.5 \mathrm{~mL})$, and the resulting solution was stirred at room temperature for $4 \mathrm{~h}$. Then, after usual workup, the products were isolated by column chromatography. The results obtained using 3a-f as activators are summarized in Table 1, which also shows the results using 1a-e for comparison. 
Table 1. Asymmetric reduction of imine $\mathbf{4 a}$ with $\mathrm{Cl}_{3} \mathrm{SiH}$ in the presence of $\mathbf{1}$ and $\mathbf{3}$

\begin{tabular}{|c|c|c|c|c|c|}
\hline entry & organic activator & $\mathrm{R}$ & yield (\%) & ee $(\%)^{\mathrm{a}}$ & config. ${ }^{b}$ \\
\hline $1^{\mathrm{c}}$ & - & - & 18 & - & - \\
\hline $2^{c}$ & $1 \mathbf{a}$ & $\mathrm{H}$ & 79 & - & - \\
\hline 3 & $1 b$ & $\mathrm{CO}_{2} \mathrm{Me}$ & 59 & 12 & $R$ \\
\hline $4^{\mathrm{c}}$ & 1c & CONHPh & 91 & 55 & $R$ \\
\hline $5^{c}$ & 1d & CONHNaph-1 & 52 & 66 & $R$ \\
\hline 6 & $1 \mathrm{e}$ & $\mathrm{CPh}_{2} \mathrm{OH}$ & 84 & 5 & $S$ \\
\hline 7 & $3 \mathbf{a}$ & $\mathrm{H}$ & 83 & - & - \\
\hline 8 & $3 b$ & $\mathrm{CO}_{2} \mathrm{Me}$ & 85 & 20 & $R$ \\
\hline 9 & $3 c$ & CONHPh & 76 & 18 & $S$ \\
\hline 10 & 3d & CONHNaph-1 & 96 & 25 & $R$ \\
\hline 11 & $3 e$ & $\mathrm{CPh}_{2} \mathrm{OH}$ & 86 & 73 & $S$ \\
\hline 12 & 3f & $\mathrm{CHPh}_{2}$ & 75 & 13 & $R$ \\
\hline
\end{tabular}

${ }^{a}$ Determined by HPLC. ${ }^{b}$ Identified by comparison of the HPLC data with literature data. ${ }^{c}$. Literature data, see ref. 7.

Although the yield of $\mathbf{5 a}$ in the absence of activators was low (entry 1), the reduction became more efficient by addition of a catalytic amount of $N$-formylpyrrolidines 1a-e (entries 2-6). Similarly, compounds 3a-f were found to work as activators for $\mathrm{Cl}_{3} \mathrm{SiH}$ (entries 7-12). Also, it was found that the highest enantioselectivity (entry 11) in 3a-f was better than that in 1a-e (entry 5). The catalytic activity of $\mathbf{3 e}$ was also checked in the reduction of a variety of imines $\mathbf{4 b}-\mathbf{h}$ and enamine $4 \mathbf{i}$ with almost similar stereoselectivity to that in the reduction of $\mathbf{4 a}$ (Table 2). On the other hand, $N$-nicotinoylpyrrolidine (3h) and $N$-(4-pyridylcarbonyl)pyrrolidine (3i) did not activate $\mathrm{Cl}_{3} \mathrm{SiH}$ (Fig. 3), suggesting an important role of a complex in which Si atom coordinates with both a nitrogen atom of picolinoyl group and a carbonyl oxygen. Little difference of \%ee between 3b-d (entries 8-10) also suggests that Si atom does not coordinate with the carbonyl group of proline ester $\mathbf{3 b}$ and amides $\mathbf{3 c}, \mathbf{d}$ but with both the nitrogen atom of picolinoyl group and the carbonyl oxygen. Furthermore, the fact that $\mathbf{3 e}$ afforded better result than 3f (Fig. 4) suggests an important role of hydroxyl group of 3e to coordinate with the $\mathrm{Si}$ atom of $\mathrm{Cl}_{3} \mathrm{SiH}$. 
Table 2. Reduction of a variety of imines $\mathbf{4 a}-\mathbf{h}$ by $\mathrm{Cl}_{3} \mathrm{SiH}$ in the presence of $\mathbf{3 e}$

Yield \% \%ee config.

${ }^{\mathrm{a}} \mathrm{N}$-Phenyl-1-( $p$-acetylphenyl)ethylamine. ${ }^{\mathrm{b}}$ Not determined.<smiles>O=C(c1ccccn1)N1CCCC1</smiles>

$3 a$

$83 \%$<smiles>O=C(c1cccnc1)N1CCCC1</smiles>

3h

$12 \%$<smiles>O=C(c1ccncc1)N1CCCC1</smiles>

3i

$21 \%$

Figure 3. Reduction of $\mathbf{4 a}$ with $\mathbf{3 a}, \mathbf{h}, \mathbf{i}$<smiles>O=C(c1ccccn1)N1CCCC1C(O)(c1ccccc1)c1ccccc1</smiles>

3e

$73 \%$ ee $((S)-5 a)$<smiles>O=C(c1ccccn1)N1CCCC1C(c1ccccc1)c1ccccc1</smiles>

3f

$13 \%$ ee $((R)-5 \mathbf{a})$

Figure 4. Reduction of $\mathbf{4 a}$ with $\mathbf{3 e , f}$ 
On the basis of these facts, we propose a mechanism shown in Figure 5, in which $\mathbf{3 e}$ coordinates with both $\mathbf{4 a}$ and $\mathrm{Cl}_{3} \mathrm{SiH}$, the transition state $\mathbf{A}$ being more likely than transition state $\mathbf{B}$, though the mechanism is a working hypothesis. ${ }^{12}$

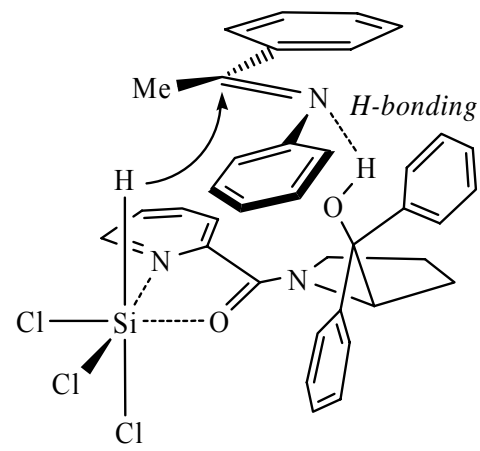

A

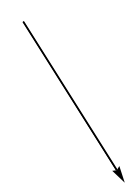

$(S)-5 \mathbf{a}$

$86.5 \%$
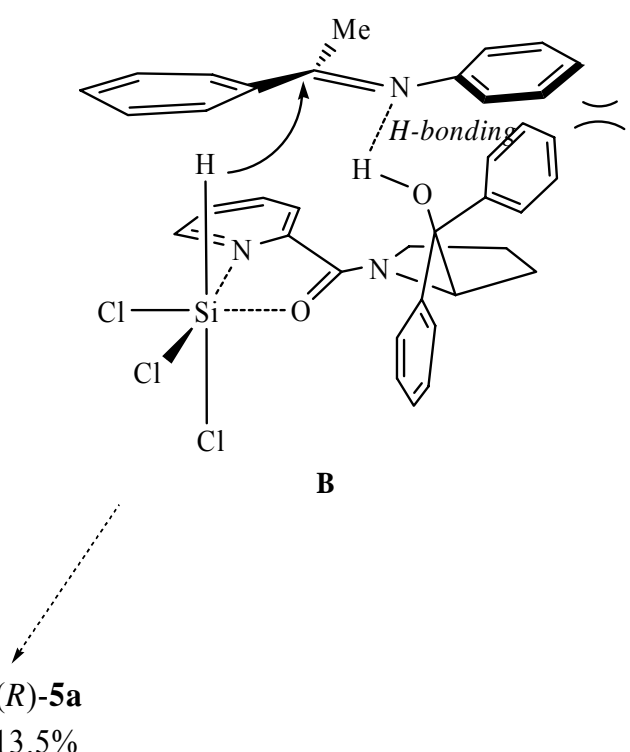

Figure 5. A plausible reaction mechanism for redution of $\mathbf{4 a}$ with $\mathbf{3 e}$

\section{Acknowledgement}

This study was supported by a Grant-in-Aid for Scientific Research on Priority Areas, (No. 420: Reaction Control of Dynamic Complexes) from the Ministry of Education, Science, Sports and Culture, Japan and by the Tokuyama Science Foundation.

\section{References and Notes}

1. Representative literatures, Borane-hydride reagents: (a) Itsuno, S.; Ito, K.; Hirao, A.; Nakahama, S. J. Chem. Soc. Chem. Commun. 1983, 469-470. (b) Corey, E. J.; Bakshi, R. K.; Shibata, S. J. Am. Chem. Soc. 1987, 109, 5551-5553. (c) Yamada, T.; Nagata, T.; Sugi, K. D.; Yorozu, K.; Ikeno, T.; Ohtsuka, Y.; Miyazaki, D.; Mukaiyama, T. Chem. Eur. J. 2003, 9, 4485-4509. Alminium-hydride reagents: (d) Yamaguchi, S.; Mosher, H. S. J. Org. Chem. 1973, 38, 1870-1877. (e) Noyori, R.; Tomino, I.; Tanimoto, Y.; Nishizawa, M. J. Am. Chem. Soc. 1984, 106, 6709-6716. Hydrogen transfer reactions: (f) Hashiguchi, S.; Fujii, A.; Takehara, J.; Ikariya, T.; Noyori, R. J. Am. Chem. Soc. 1995, 117, 7562-7563. (g) Uematsu, N.; Fujii, A.; Hashiguchi, S.; Ikariya, T.; Noyori, R. J. Am. Chem. Soc. 1996, 118, 2521-2522. Hydrogenation: (h) Ohkuma, T; Ooka, H.; Hashiguchi, S.; Ikariya, T.; Noyori, R. J. Am. Chem. Soc. 1995, 117, 2675-2676. (i) Ohkuma, T.; Koizumi, M.; Doucet, H.; Pham, T.; 
Kozawa, M.; Murata, K.; Katayama, E.; Yokozawa, T.; Ikariya, T.; Noyori, R. J. Am. Chem. Soc. 1998, 120, 13529-13530. (j) Ohkuma, T.; Sandoval, C. A.; Srinivasan, R.; Lin, Q.; Wei, Y.; Muniz, K.; Noyori, R. J. Am. Chem. Soc. 2005, 127, 8288-8289.

2. Representative literatures, Hydride reagents: (a) Langlois, N.; Dang, T.-P.; Kagan, H. B. Tetrahedron Lett. 1973, 4865-4868. (b) Itsuno, S.; Nakano, M.; Ito, K.; Hirao, A.; Owa, M.; Kanda, N.; Nakahama, S. J. Chem. Soc. Parkin. Trans. 1 1985, 2615-2619. (c) Becker, R.; Brunner, H.; Mahboobi, S.; Wiegrebe, W. Angew. Chem. Int. Ed. 1985, 24, 995-996. (d) Verdaguer, X.; Lange, U. E. W.; Reding, M. T.; Buchwald, S. L. J. Am. Chem. Soc. 1996, 118, 6784-6789. (e) Nolin, K.A.; Ahn, R. W.; Toste, F. D. J. Am. Chem. Soc. 2005, 127, 12462-12463. Hydrogen transfer reactions: (f) Uematsu, N.; Fujii, A.; Hashiguchi, S.; Ikariya, T.; Noyori, R. J. Am. Chem. Soc. 1996, 118, 4916-4917. (g) Mao, J.; Baker, D. C. Org. Lett. 1999, 1, 841-843. Hydrogenation: (h) Levi, A.; Modena, G.; Scorrano, G. J. Chem. Soc. Chem. Commun. 1975, 6-7. (i) Burk, M. J.; Feaster, J. E. J. Am. Chem. Soc. 1992, 114, 6266-6267. (j) Willoughpy, C. A.; Buchwald, S. L. J. Am. Chem. Soc. 1994, 116, 8952-8965. (k) Trifonova, A.; Diesen, J. S.; Chapman, C. J.; Abdersson, P. G. Org.Lett. 2004, 6, 3825-3827. (1) Solinas, M.; Pfaltz, A.; Cozzi, P. G.; Leiner, W. J. Am. Chem. Soc. 2004, 126, 16142-16147. (n) Moessner, C.; Bolm, C.; Angew. Chem. Int. Ed. 2005, 44, 7564-7567.

3. Some recent reviews, see: (a) Noyori, R.; Hashiguchi, S. Acc. Chem. Res. 1997, 30, 97-102. (b) Corey, E. J.; Helal, C. J. Angew. Chem. Int. Ed. 1998, 37, 1986-2012. (c) Noyori, R.; Ohkuma, T. Angew. Chem. Int. Ed. 2001, 40, 40-73. (d) Carpentier, J.-F.; Bette, V. Curr. Org. Chem. 2002, 6, 913-936. (e) Riant, O.; Mostefai, N.; Courmarcel, J. Synthesis 2004, 2943-2958. (f) Tararov, V. I.; Börner, A. Synlett 2005, 203-211. (g) Weinreb, S. M.; Orr, R. K. Synthesis 2005, 1205-1227.

4. (a) Pirkle, W. H.; Hauske, J. R. J. Org. Chem. 1977, 42, 2781-2782. (b) Benkeser, R. A.; Snyder, D. C. J. Oganomet. Chem. 1982, 225, 107-115. (c) Akutagawa, S. J. Synth. Org. Chem. Jpn. 1986, 44, 513-518. (d) Okamoto, H.; Kato, S. Bull. Chem. Soc. Jpn. 1991, 64, 2128-2130. (e) Zulehler, W.; Neure, B.; Rau, G. Ullmann's Encyclopedia of Industrial Chemistry; VCH: Weinheim, 1993; Vol. A23, 721-741. (f) Chong, P. Y.; Janicki, S. Z.; Petillo, P. A. J. Org. Chem. 1998, 63, 8515-8521. (g) Enholm, E. J.; Schulte II, J. P. J. Org. Chem. 1999, 64, 2610-2611. (h) Iwasaki, K.; Nozawa, S. J. Synth. Org. Chem. Jpn. 2000, 58, 62-64. (i) Hayashi, T.; Hirata, S.; Kitayama, K.; Tsuji, H.; Torii, A.; Uozumi, Y. J. Org. Chem. 2001, 66, 1441-1449. (j) Cheng, C.-H.; Shih, H.-H.; Shih, H.-T. Org. Lett. 2001, 3, 811-814. (k) Choi, S.-B.; Kim, B.-K.; Boudjouk, P.; Grier, D. G. J. Am. Chem. Soc. 2001, 123, 8117-8118. (1) Iwasaki, F. Oda, J. Synth. Org. Chem. Jpn. 2001, 59, 1005-1007.

5. Kobayashi, S.; Yasuda, M.; Hachiya, I. Chem. Lett. 1996, 407-408. 
6. Iwasaki, F.; Onomura, O.; Mishima, K.; Maki, T.; Matsumura, Y. Tetrahedron Lett. 1999, 40, 7507-7511.

7. Iwasaki, F.; Onomura, O.; Mishima, K.; Kanematsu, T.; Maki, T.; Matsumura, Y. Tetrahedron Lett. 2001, 42, 2525-2527.

8. Malkov, A. V.; Stončius, S.; MacDougall, K. N.; Mariani, A.; McGeoch, G. D.; Kočovský, P. Tetrahedron Lett. 2006, 62, 264-284.

9. Pyridine catalyzed hydrosilylation of unsaturated carbon-carbon bonds with $\mathrm{Cl}_{3} \mathrm{SiH}$, see. (a) Nozakura, S.; Konotsune, S. Bull. Chem. Soc. Jpn. 1956, 29, 322-326. (b) Pike, R. A. J. Org. Chem. 1962, 27, 2186-2190. Also pyridine promoted reduction of phosphine oxides with $\mathrm{Cl}_{3} \mathrm{SiH}$, see. (c) Horner, L.; Balzer, W. D. Tetrahedron Lett. 1965, 1157-1162. Reductive silylation of carbonyl compounds proceeded using $\mathrm{Cl}_{3} \mathrm{SiH}$-tertiary amine afforded not alcohols but alkylsilanes, see; (d) Benkeser, R. A.; Smith, W. E. J. Am. Chem. Soc. 1969, 91, 1556-1557.

10. Recently, we disclosed that using $N$-picolinoyl-L-proline derivative $\mathbf{3 b}$ as an activator for $\mathrm{Cl}_{3} \mathrm{SiH}$ reduced ketones to chiral alcohols with good optical purities, see ref. 13. And more recently, other group reported that 2-pyridyloxazoline $\mathbf{6}$ worked well as an efficient activator for $\mathrm{Cl}_{3} \mathrm{SiH}$ to reduce ketones and imines, see ref. 14 .

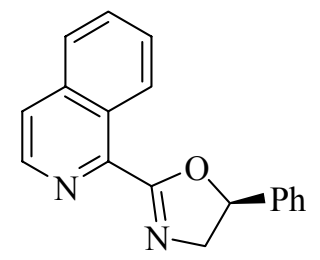

6

11. Some recent literatures concerning organic activators for other trichlorosilanes, see: (a) Nakajima, M.; Saito, M.; Shiro, M.; Hashimoto, S. J. Am. Chem. Soc. 1998, 120, 6419-6420. (b) Denmark, S. E.; Su, X; Nishigaichi, Y. J. Am. Chem. Soc. 1998, 120, 12990-12991. (c) Shimada, T.; Kina, A.; Ikeda, S.; Hayashi, T. Org. Lett. 2002, 4, 2799-2801. (d) Denmark, S. Fan, Y. J. Am. Chem. Soc. 2002, 124, 4233-4235. (e) Wong, W-L.; Lee, C-S.; Leung, H-K.; Kwong, H.-L. Org. Biomol. Chem. 2004, 2, 1967-1969. (f) Ogawa, C.; Sugiura, M.; Kobayashi, S. Angew. Chem. Int. Ed. 2004, 43, 6491-6493. (g) Nakajima, M.; Kotani, S.; Ishiduka, T.; Hashimoto, S. Tetrahedron Lett. 2005, 46, 157-159.

12. Intermediates such as one involving a Si-O bond can not be ruled out.

13. Matsumura, Y.; Onomura, O.; Iwasaki, F. Jpn. Kokai Tokkyo Koho (2005), JP 2005029503: CA 142:176534 (2005).

14. Malkov, A. V.; Stewart Liddon, A. J. P.; Ramírez-López, P.; Bendová, L.; Haigh, D.; Kočovský, P. Angew. Chem. Int. Ed. 2006, 45, 1432-1435. 